\title{
Ensino de programação: um estudo preliminar nos cursos de Licenciatura em Computação no Brasil
}

\author{
Lucas Pedroso Krzyzanowski', Carlos Roberto Beleti Junior', Robertino Mendes \\ Santiago Junior', Raimundo Alberto Tostes' \\ Universidade Federal do Paraná (UFPR) \\ 86.900-000 - Jandaia do Sul - PR - Brasil \\ lucaskrzy@gmail.com, \{carlosbeleti,robertino, tostes\}@ufpr.br
}

\begin{abstract}
This paper presents a preliminary study of undergraduate degree courses in the field of Computer Science in Brazil, analyzing the introductory disciplines of Algorithms and Programming and its curricular components, as well as the approval, fail and evasion rates to understand the adversities encountered by students. A search was made for the information and pedagogical projects of the courses, and specifically for two UFPR courses, their introductory programming subjects, analyzing the failure rates in the subject and their connection with student dropout. It is hoped to deepen the research to all courses of this area in Brazil, to analyze the correlation of introductory programming disciplines with dropout in them.
\end{abstract}

Resumo. Este artigo apresenta um estudo preliminar dos cursos de Licenciatura na área da Computação no Brasil, a fim de analisar as disciplinas introdutórias de Algoritmos e Programação e suas ementas, além dos dados de aprovação, reprovação e evasão para compreender as adversidades encontradas pelos estudantes. Foi realizada uma busca pelas informações e projetos pedagógicos dos cursos, e especificamente para dois cursos da UFPR, suas disciplinas introdutórias de programação, analisando os índices de reprovação na disciplina e sua ligação com a evasão de alunos. Almeja-se aprofundar a pesquisa em todos os cursos dessa área no Brasil, para analisar a correlação das disciplinas introdutórias de programação com a evasão nos mesmos.

\section{Introdução}

O mercado de trabalho na área das Tecnologias da Informação e Comunicação no Brasil é o $7^{\circ}$ maior do mundo sendo responsável por $7 \%$ do PIB nacional, e mesmo sendo um mercado tão promissor, existem índices de falta mão de obra qualificada [Brasscom 2019].

Essa grande demanda de profissionais qualificados gera a necessidade de capacitação profissional. Neste sentido, segundo a Sociedade Brasileira de Computação [SBC 2002] o curso de Licenciatura em Computação tem como intuito formar um profissional docente que incorpore múltiplas competências, sendo elas: saberes e habilidades de criatividade e inovação, de cooperação e de trabalho em equipe, de gestão e tomada de decisões, de aquisição e produção de conhecimentos, de expressão e comunicação, não sendo somente reprodutor de conhecimentos já estabelecidos.

Os cursos de Licenciatura na área da Computação, bem como demais cursos da área de Exatas, tais como em cursos de Engenharia, têm apresentado dificuldades nas disciplinas 
VIII Congresso Brasileiro de Informática na Educação (CBIE 2019)

Anais dos Workshops do VIII Congresso Brasileiro de Informática na Educação (WCBIE 2019)

introdutórias de Algoritmos e Programação [Giraffa and Mora 2013] [Hoed 2016] [Dejavite et al. 2017].

Segundo [Koliver, Dorneles and Casa 2004], um dos principais motivos pela evasão em disciplinas referentes ao ensino de algoritmos é o despreparo dos estudantes para enfrentar uma disciplina, cujo objetivo principal é auxiliar no desenvolvimento da habilidade de solucionar problemas utilizando raciocínio lógico.

Com o exposto, este trabalho tem como objetivo demonstrar um estudo prévio sobre os cursos de Licenciatura na área da Computação em vigência no Brasil, em que, por meio do estudo dos projetos pedagógicos dos cursos foram analisadas as disciplinas introdutórias de Algoritmos e Programação, observando inicialmente, os conteúdos mais abordados nas disciplinas, assim como os índices de aprovação, reprovação e evasão nas mesmas e observando seu impacto no grau de evasão nos cursos de Licenciatura em Computação da Universidade Federal do Paraná (UFPR), no período de 2015 a 2018.

\section{Trabalhos relacionados}

Os elevados índices de evasão e as dificuldades enfrentadas por alunos em cursos superiores têm sido objeto de estudo de diversos trabalhos nos últimos anos. Sendo assim, foram pesquisados trabalhos que reforçam esta afirmação, buscando por pesquisas que também têm interesse em contribuir com o ensino e aprendizagem de Algoritmos e Programação ou que se preocupam se tais áreas têm relação com os altos níveis de reprovação e evasão no ensino superior [Reino et al. 2015], [Damasceno and Carneiro 2018], [Dejavite et al. 2017], [Piva and Freitas 2010].

No trabalho de [Galafassi, Gluz and Galafassi 2013] utilizou-se da plataforma eMEC $^{1}$ para pesquisar por Instituições de Ensino Superior (IES), e em seguida foram analisados os Ambientes Virtuais de Aprendizagem (AVA) utilizados pelas mesmas como ferramenta de suporte à aprendizagem.

Trabalhos como os desenvolvidos por [Reino et al. 2015] e [Damasceno and Carneiro 2018], buscam identificar as principais causas das altas taxas de evasão em cursos superiores buscando assim, propor melhorias que visam reduzi-las. No primeiro, foi realizada uma análise de evasão no curso de bacharelado em Sistemas de Informação da Universidade Federal de Alagoas, na modalidade de Educação a Distância, constatando-se que 83 \% dos alunos evadiram do curso e dentre estes, que responderam o questionário aplicado, $84 \%$ evadiram nos três primeiros semestres. Já no segundo, foi apresentado um estudo relacionado à evasão no curso de Bacharelado em Sistemas da Informação ofertado pela Universidade Federal de Uberlândia, campus Santa Mônica, referente aos anos de 2009 a 2013. Nesse estudo foi constatado que a turma com maior evasão chegou a $72 \%$ dos alunos evadidos e a com menor evasão chegou a $43 \%$, além do elevado número de alunos retidos que não concluíram o curso no prazo regular.

Além da área da Computação, outros estudos apontam que cursos como as Engenharias e em áreas da Matemática e Física também sofrem com as altas taxas de reprovação e evasão. $O$ trabalho de [Dejavite et al. 2017] apresenta um estudo referente às disciplinas de Algoritmos e Programação nos cursos de Engenharia ofertados pela Universidade Federal do Paraná, mostrando as taxas de aprovação, assim como, uma análise dos tópicos estruturais mais abordados nas ementas. [Stadelhofer and Gasparini 2018],

\footnotetext{
${ }^{1}$ Cadastro Nacional de Cursos e Instituições de Educação Superior - http://emec.mec.gov.br/
} 
sugeriram uma relação dos conteúdos estudados na disciplina com o curso de formação dos estudantes, em que, por meio de um mapeamento sistemático, foi observado que a taxa de aprovação dos alunos quando utilizada uma abordagem contextualizada apresentou uma melhoria significativa.

Em seu trabalho de conclusão de curso, [Hoed 2016] apresentou uma análise na evasão em cursos superiores da área da Computação utilizando-se de dados do Instituto Nacional de Estudos e Pesquisas Educacionais Anísio Teixeira $\left(\mathrm{INEP}^{2}\right)$ em que conseguiu identificar motivos que levaram aos elevados níveis de evasão nos cursos, tais como, o gênero dos estudantes, ser cotista ou não, e nota de corte de entrada no curso.

Segundo [Florencio 2015] algumas disciplinas acabam atuando diretamente no grau de evasão de alunos, dentre elas a disciplina de Computação Básica, que é uma disciplina ofertada pelo Departamento de Ciências da Computação (CIC) e não possui nenhum prérequisito. Essa disciplina apresenta uma taxa de reprovação de $41 \%$ e afeta diretamente a evasão no curso, pois 89 \% dos alunos que reprovam em Computação Básica evadem.

Segundo [Girafa e Mora 2013], algumas das principais dificuldades encontradas pelos discentes nas disciplinas de Algoritmos e Programação são: expressão em língua materna (escrita), interpretação de textos e enunciados, hábitos de estudo e pesquisa e, especialmente, com formação básica deficitária no que tange a conteúdos de Matemática, impactando diretamente nos índices de evasão. Conforme [Piva Jr. and Freitas 2010] a dificuldade encontrada por alunos que não possuem uma boa base nas disciplinas de português e matemática impacta diretamente seu desempenho, levando-os a terem dificuldades na abstração dos conteúdos ensinados, desmotivando-os, causando assim a evasão ou mudança de curso.

É provável que muitas variáveis relacionadas ao desempenho acadêmico estejam fortemente vinculadas a um contexto socioeconômico e cultural mais amplo. Ou seja, o desempenho acadêmico também reflete as contradições da sociedade, de uma maneira geral, e a desigualdade social de forma bem particular, conforme apontam [Veloso and Almeida 2013] [Hoed 2016].

\section{Metodologia}

A metodologia utilizada no trabalho é dividida em quatro fases sendo elas: 1) levantamento dos dados do INEP referentes aos índices de evasão e conclusão dos cursos; 2) coleta de dados dos cursos na plataforma e-MEC; 3) estudo preliminar das disciplinas de Algoritmos e Programação nos Projetos Pedagógicos dos Cursos (PPCs); 4) análise dos dados de reprovação e evasão nas disciplinas introdutórias relacionadas a Algoritmos e Programação nos cursos de Licenciatura em Computação na UFPR.

1. Coleta de dados no INEP: conforme observado em referenciais na literatura, cursos da área de Exatas e Computação historicamente apresentam índices elevados de evasão, assim buscou-se nessa plataforma informações sobre as taxas de permanência, conclusão e evasão dos cursos de Licenciatura na área de Computação no país.

2. Coleta de dados na plataforma e-MEC: por meio dos dados obtidos na plataforma, foi possível a realização de análises como distribuições geográficas, modalidade

\footnotetext{
2 Instituto Nacional de Estudos e Pesquisas Educacionais Anísio Teixeira - http://www.inep.gov.br/
} 
VIII Congresso Brasileiro de Informática na Educação (CBIE 2019)

Anais dos Workshops do VIII Congresso Brasileiro de Informática na Educação (WCBIE 2019)

(presencial ou EaD) e de gratuidade dos cursos de Licenciatura na área de Computação no Brasil. Além disso, os dados obtidos por meio do sistema possibilitaram uma filtragem dos cursos ainda ativos no Brasil, onde foram selecionados aqueles com criação anterior ou igual ao ano de 2014, caracterizados como gratuitos e com Conceito de Curso (CC) superior ou igual a três.

3. Estudo dos PPCs: com o escopo de cursos definidos foram estudadas as disciplinas introdutórias de Algoritmos e Programação contidas nos PPCs dos cursos elencados, sendo analisados os componentes curriculares destas.

4. Dados da UFPR: foram levantados os dados de aprovação, reprovação e desistência, no período de 2015 a 2018, dos cursos de Licenciatura em Computação da UFPR, ofertados no campus Avançado em Jandaia do Sul e no Setor Palotina.

\section{Resultados preliminares}

Nesta seção são apresentados os dados obtidos nos portais governamentais do INEP e eMEC, além das informações coletadas sobre os cursos selecionados como, por exemplo, das ementas de disciplinas relacionadas à Algoritmos e Programação de seus projetos pedagógicos de curso (PPCs).

\subsection{Panorama geral}

Nos indicadores educacionais dos cursos de graduação referente aos anos 2010-2015 apresentados pelo INEP [Brasil 2015] foram encontrados 55 cursos que possuem nomes relacionados à Computação ou Informática e que estão classificados como área Educação segundo a Organização para a Cooperação e Desenvolvimento Econômico (OCDE). A Tabela 1 exibe as taxas de permanência, conclusão e desistência acumuladas dos cursos.

Com as informações da Tabela 1, pode-se observar que, no período da consulta, pouco mais da metade dos alunos que iniciaram no curso permaneceram no mesmo. Ademais, apenas 9,2 \% dos estudantes concluíram suas formações e 38,7 \% acabaram desistindo do curso.

Tabela 1. Dados INEP indicadores de trajetória anos 2010-2015

\begin{tabular}{c|c|c}
\hline $\begin{array}{c}\text { Taxa de Permanência } \\
\text { (\%) }\end{array}$ & Taxa de Conclusão (\%) & Taxa de Desistência (\%) \\
\hline 52,1 & 9,2 & 38,7 \\
\hline
\end{tabular}

FONTE: Adaptado de BRASIL (2015)

Em pesquisa no portal e-MEC [Brasil 2019], foi constatado que existem atualmente 169 cursos de Licenciatura na área de Computação e, dentre estes, 127 ainda estão ativos, sendo distribuídos conforme apresentado na Tabela 2. Dentre os cursos encontrados foram aplicados critérios de seleção, buscando por aqueles que atenderam os seguintes requisitos: gratuito, com criação inferior ou igual ao ano de 2014, conceito de curso (CC) maior ou igual a 3, e dados do curso disponíveis em sites institucionais. Com o filtro aplicado foram encontrados 26 cursos de modalidade Presencial e 1 curso da modalidade EAD. 
VIII Congresso Brasileiro de Informática na Educação (CBIE 2019)

Anais dos Workshops do VIII Congresso Brasileiro de Informática na Educação (WCBIE 2019)

Tabela 2. Distribuição dos cursos ativos

\begin{tabular}{c|c|c}
\hline \multirow{2}{*}{ Categoria } & \multicolumn{2}{|c}{ Modalidade } \\
\cline { 2 - 3 } & Presencial & EAD \\
\hline Gratuito & 67 & 21 \\
\hline Não gratuito & 30 & 9 \\
\hline
\end{tabular}

É importante ressaltar as divergências encontradas nas informações buscadas na plataforma e-MEC e no site do INEP. Nos dados disponibilizados pelo INEP foram consideradas as informações disponíveis entre 2010 a 2015 e referentes à classificação da OCDE grande área do curso "educação" e nome da área "Formação de professor de computação (informática) ", com isso foram encontrados os cursos: Ciência da Computação, Ciências da Computação, Computação, Computação e Informática e Informática. Enquanto os dados encontrados na plataforma e-MEC foram obtidos por meio de consultas avançadas, buscando por cursos de graduação que atendessem os seguintes critérios: classificados com grau Licenciatura, no campo de Curso foram buscados utilizando as palavras "Computação" e "Informática", além de no campo gratuidade foram realizadas buscas referentes aos cursos gratuitos ou não.

\subsection{Informações geográficas}

A partir dos dados obtidos na plataforma e-MEC foi possível realizar uma busca das instituições cadastradas no sistema, possibilitando assim a distribuição geográfica dos cursos (Tabela 3), além da pesquisa mais aprofundada nos cursos que serão estudados nos próximos tópicos.

Tabela 3. Distribuição geográfica dos cursos por categoria

\begin{tabular}{c|c|c|c|c|c|c}
\hline \multirow{2}{*}{ Categoria } & \multirow{2}{*}{ Modalidade } & \multicolumn{5}{|c}{ Região } \\
\cline { 3 - 7 } & & Norte & Nordeste & Centro-Oeste & Sudeste & Sul \\
\hline \multirow{2}{*}{ Gratuito } & Presencial & 20 & 30 & 8 & 2 & 7 \\
\cline { 2 - 7 } & EAD & 2 & 11 & 4 & 1 & 3 \\
\hline \multirow{2}{*}{$\begin{array}{c}\text { Não } \\
\text { gratuito }\end{array}$} & Presencial & 1 & 1 & 5 & 6 & 17 \\
\cline { 2 - 7 } & EAD & 0 & 2 & 1 & 1 & 5 \\
\hline
\end{tabular}

Dentre os dados apresentados, foram selecionados para estudo conforme critérios já descritos anteriormente 27 cursos, sendo eles distribuídos em: 1 curso EAD com sede na região norte e 26 cursos presenciais, estando esses distribuídos da seguinte forma: 5 na região norte, 13 nordeste, 4 centro-oeste e 4 sul.

\subsection{PPCS e Componentes curriculares}

Após conseguir acesso aos PPCs de cada curso selecionado, foi possível analisar as componentes curriculares, buscando as disciplinas introdutórias de Algoritmos e Programação e registrando os conteúdos ministrados assim como sua carga horária. Dentre as disciplinas estudadas foi constatado que suas cargas horárias, em média, estão entre $51 \mathrm{e}$ 70 horas (38\%), 71 e 90 horas (46\%), aparecendo em menor quantidade com $12 \%$ e $4 \%$ representando, respectivamente, carga horária menor que 50 horas e maior que 90 horas.

Os tópicos curriculares que mais apareceram nas ementas das disciplinas foram: introdução à disciplina, pseudocódigo, linguagem de programação estruturada, operadores 
VIII Congresso Brasileiro de Informática na Educação (CBIE 2019)

Anais dos Workshops do VIII Congresso Brasileiro de Informática na Educação (WCBIE 2019)

lógicos e matemáticos, estruturas de decisão e de repetição, vetores e matrizes, funções e ponteiros. A Tabela 4 apresenta o percentual de cada tópico curricular nos cursos identificados.

Tabela 4. Tópicos Curriculares nas primeiras disciplinas com contato a programação

\begin{tabular}{c|c}
\hline Tópicos Curriculares & Ementas que contemplam o tópico (\%) \\
\hline Introdução à disciplina & 93 \\
\hline Pseudocódigo & 37 \\
\hline Linguagem de programação estruturada & 100 \\
\hline Operadores lógicos e matemáticos & 100 \\
\hline Estruturas de decisão e de repetição & 96 \\
\hline Vetores e matrizes & 74 \\
\hline Funções & 41 \\
\hline Ponteiros & 19 \\
\hline
\end{tabular}

Conforme apontado na Tabela 4, constata-se que a maioria dos cursos contempla em suas ementas das disciplinas introdutórias de Algoritmos e Programação, tópicos de Introdução à disciplina (93\%), Linguagem de programação estruturada (100\%), Operadores lógicos e matemáticos (100\%), Estruturas de decisão e de repetição (96 \%), o que reforça esses como tópicos essenciais às disciplinas. Vetores e matrizes também são conceitos importante e a maioria dos cursos contempla, porém em percentual ligeiramente menor (74 \%). Funções e ponteiros aparecem em menor percentual, representando, respectivamente, 41 e $19 \%$.

Quanto às componentes curriculares das disciplinas, o trabalho de [Dejavite et al. 2017] mostrou que em disciplinas de Algoritmos e Programação para cursos de Engenharia, também há uma falta de padronização dos conteúdos nas ementas disciplinares, de forma semelhante ao levantamento realizado neste trabalho. Assim, surgem questionamentos, tais como: "por que alguns tópicos disciplinares são abordados apenas em algumas disciplinas?", ou ainda, "qual a importância de cada tópico para a disciplina introdutória de Algoritmos e Programação em um curso de Computação?".

\subsection{Análise preliminar na instituição UFPR}

Com informações do Sistema de Informações para o Ensino (SIE) $)^{3}$ e indicadores da PROGRAD $^{4}$ da UFPR foram obtidos os dados de aprovação e reprovação dos alunos dos cursos de Licenciatura em Computação da UFPR, ofertados no Campus Avançado em Jandaia do Sul e no Setor Palotina. Com essas informações, foram confirmados os elevados níveis de reprovação na disciplina referente ao primeiro contato com o ensino de Algoritmos e Programação, constatando que, assim como o trabalho realizado por [Florencio 2015], a

\footnotetext{
${ }^{3}$ https://www.cce.ufpr.br/portal/suporte-sie/

${ }^{4}$ http://indicadores.ufpr.br/ind/index.php
} 
VIII Congresso Brasileiro de Informática na Educação (CBIE 2019)

Anais dos Workshops do VIII Congresso Brasileiro de Informática na Educação (WCBIE 2019)

disciplina de Algoritmos pode ser um elemento essencial no que se refere a evasão dos alunos.

Na Figura 1 encontram-se os dados de aprovação, reprovação por nota, reprovação por frequência e cancelamento dos estudantes do curso de Licenciatura em Computação (LC) do Campus Avançado em Jandaia do Sul entre os anos de 2015 e 2018. Assim como encontrado nos trabalhos de [Dejavite et al. 2017] e [Koliver et al. 2004] foram confirmados os elevados índices de reprovação nas disciplinas de Algoritmos e Programação seja por nota ou frequência.

Além das informações referentes à aprovação e reprovação, buscou-se, em um primeiro momento analisar os dados de evasão do curso de LC em Jandaia do Sul. Nessa análise, constatou-se que, em média, $62,5 \%$ dos alunos que reprovaram na primeira disciplina de Algoritmos e Programação por nota e $70 \%$ dos que reprovaram por frequência acabaram trancando o curso (Figura 2). É interessante destacar que, em média, metade dos alunos que conseguem aprovação nessa disciplina continuam mantêm-se matriculado no curso, o que mostra que, assim como apontado no trabalho de [Florencio 2015] essas disciplinas têm fundamental importância na formação do estudante na área da Computação.

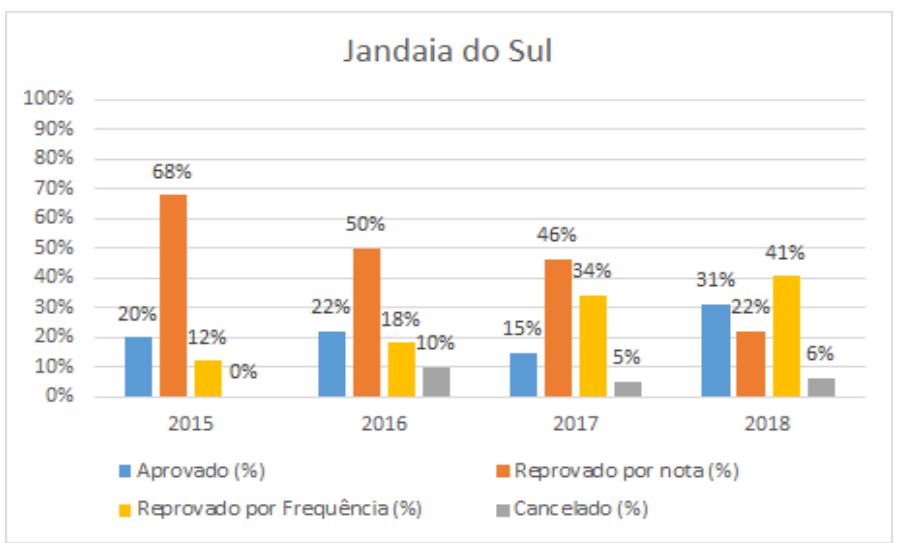

Figura 1. Análise de aprovação na disciplina de Algoritmos UFPR Campus Avançado em Jandaia do Sul

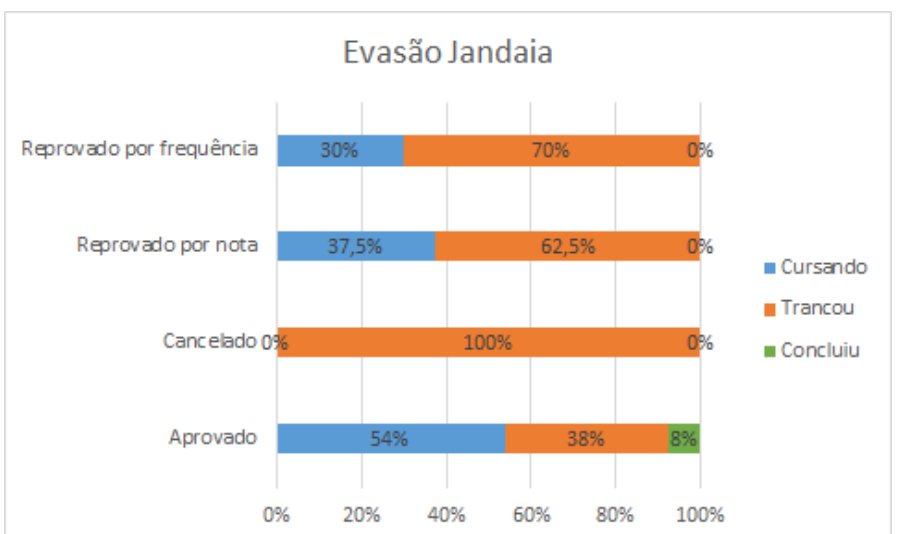

Figura 2. Análise de evasão conforme aprovação na disciplina de Algoritmos UFPR Campus Avançado em Jandaia do Sul

Assim como no Campus Avançado em Jandaia do Sul, os dados de reprovação sejam por nota ou por frequência no Setor Palotina são expressivos, conforme observado na Figura 3, chegando em média a $24,25 \%$ de reprovações por nota e $51,75 \%$ de reprovações 
VIII Congresso Brasileiro de Informática na Educação (CBIE 2019)

Anais dos Workshops do VIII Congresso Brasileiro de Informática na Educação (WCBIE 2019)

por frequência, indo ao encontro dos resultados de trabalhos correlatos presentes na literatura [Dejavite et al. 2017] [Koliver et al. 2004].

De forma semelhante ao ocorrido no Campus Avançado em Jandaia do Sul, no Setor Palotina o percentual de evasão é elevado. Um dado alarmante foi a quantidade de concluintes, pois no Setor não houve nenhum aluno que concluiu o curso no período estabelecido neste trabalho, conforme observado na Figura 4.

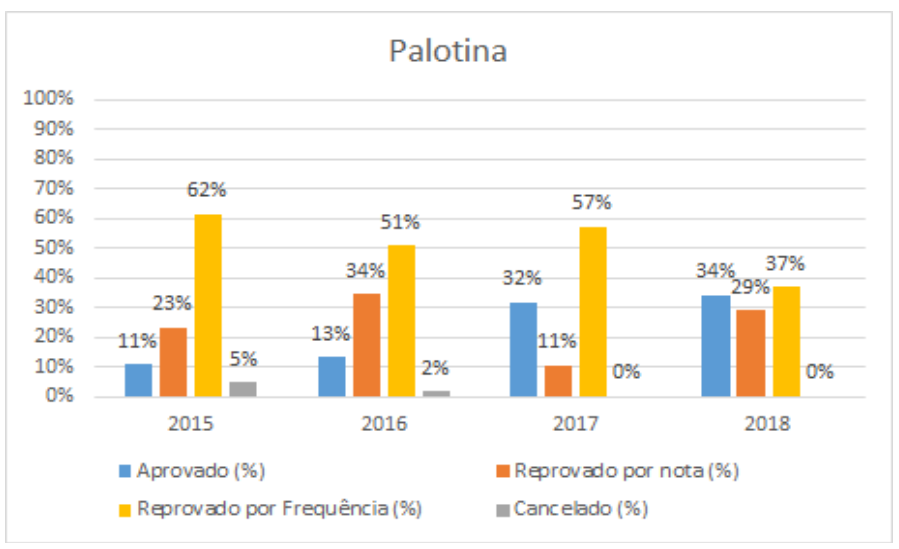

Figura 3. Análise de aprovação na disciplina de Algoritmos UFPR Setor Palotina

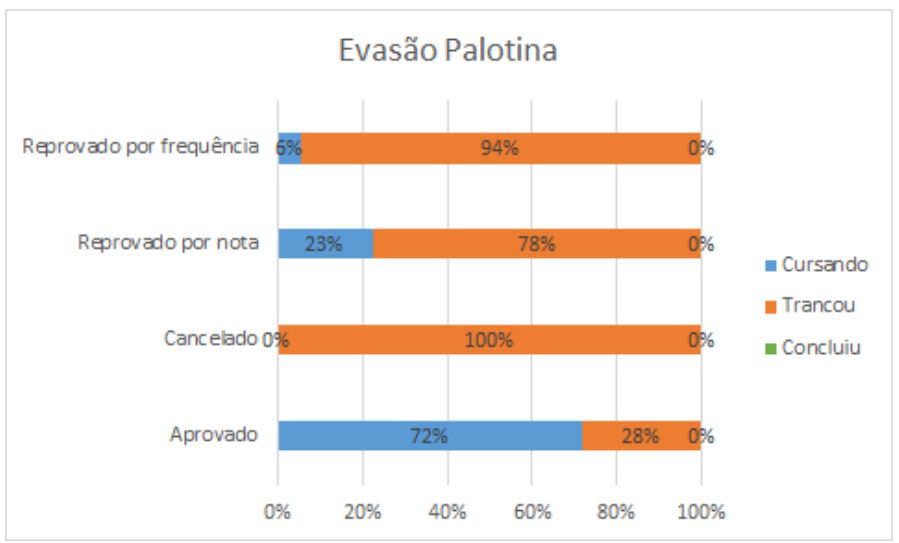

Figura 4. Análise de evasão conforme aprovação na disciplina de Algoritmos UFPR Setor Palotina

\section{Considerações finais}

Este trabalho foi motivado pela falta de informações sobre as razões que possam explicar as altas taxas de reprovação nas disciplinas de primeiro contato com o ensino de Algoritmos e Programação e evasão nos cursos de Licenciatura em Computação no Brasil.

$\mathrm{O}$ artigo em tela teve como objetivo demonstrar parcialmente a pesquisa que está sendo desenvolvida no trabalho de conclusão de curso de um Licenciando em Computação do Campus Avançado em Jandaia do Sul. Preliminarmente foi possível constatar que: 1) As taxas de reprovação e evasão nos cursos de Licenciatura na área da Computação são tão elevadas quanto em cursos da área de Exatas [Dejavite et al. 2017] [Stadelhofer, \& Gasparini 2018]; 2) Existem 169 cursos de Licenciatura na área da Computação no Brasil catalogadas na plataforma e-MEC, porém apenas 127 em situação ativa, e 27 com CC maior ou igual a 3 
VIII Congresso Brasileiro de Informática na Educação (CBIE 2019)

Anais dos Workshops do VIII Congresso Brasileiro de Informática na Educação (WCBIE 2019)

e com informações disponíveis em suas páginas institucionais, o que aponta a necessidade de que, além da criação de diversos cursos, deve-se assegurar que tais possuam condições de funcionamento e portais virtuais atualizados com suas informações; 3) que os cursos presenciais e gratuitos de Licenciatura na área da Computação aparecem em sua maioria nas regiões Norte e Nordeste enquanto que os não gratuitos nas regiões Sul e Sudeste; 4) divergências nas matrizes curriculares dos cursos; 5) que os campi da UFPR no interior também apresentam dificuldades quanto aos índices de reprovação e evasão das disciplinas e cursos, respectivamente.

Relevante destacar informações como: a taxa de escolarização líquida da população brasileira de 18 a 24 anos que é baixa: 14,4 \%, segundo o Censo da Educação Superior de 2017 , e que cerca de 75,3 \% de todas as matrículas de graduação estão no setor privado e 24,7\% no setor público [Brasil 2017]. Ainda segundo [Barros 2015] há uma flagrante assimetria na oferta de cursos superiores nas áreas que requerem mais investimento em infraestrutura física, especialmente laboratorial, havendo, portanto, uma grande concentração de cursos na área de Ciências Sociais Aplicadas (administração, direito, contabilidade, negócios), cerca de $41,6 \%$ do total de matrículas, mormente nas IES privadas. As áreas que requerem maiores investimentos, como as que tendem a ser majoritariamente atendidas pelo setor público, o que implica na impossibilidade do atendimento da demanda. No caso do norte e nordeste, é razoável inferir, portanto, que os investimentos em cursos nas áreas de computação não suprem as condições mercadológicas, reduzindo-se a uma questão econômica em detrimento de uma visão plena de educação como desenvolvimento.

Destaca-se que, como continuação deste trabalho, serão realizadas entrevistas com os coordenadores dos cursos selecionados e professores das disciplinas elencadas, com o intuito de observar quais as maiores dificuldades encontradas pelos alunos nessas disciplinas iniciais, buscando assim reduzir os números de reprovação e evasão na disciplina e no curso.

Além das entrevistas serão realizadas outras atividades buscando maior embasamento para ações pedagógicas. Atividades como:

- Quantificar os dados de aprovação, reprovação e evasão nas disciplinas iniciais de Algoritmos e Programação nos cursos cujos PPCs foram elencados;

- Analisar o impacto de informações socioeconômicas dos alunos que cursam tais cursos de graduação;

- Analisar o impacto dos componentes curriculares nas taxas de evasão e reprovação;

- Propor melhorias no ensino de algoritmos e programação de computadores.

\section{Referências}

Barros, A.S.X. (2015) Expansão da Educação Superior no Brasil: Limites e Possibilidades. Revista Educação e Sociedade, 36(131) 361-390.

Brasil (2015). Instituto Nacional de Estudos e Pesquisas Educacionais Anísio Teixeira. Indicadores Educacionais. Disponível em: $<$ http://portal.inep.gov.br/web/guest/indicadores-educacionais $>$. Acesso em: 13 de maio de 2019.

Brasil (2017). Instituto Nacional de Estudos e Pesquisas Educacionais Anísio Teixeira. Censo da Educação Superior 2017. Disponível em: <http://inep.gov.br/censo-daeducacao-superior>. Acesso em: 26 de junho de 2019. 
VIII Congresso Brasileiro de Informática na Educação (CBIE 2019)

Anais dos Workshops do VIII Congresso Brasileiro de Informática na Educação (WCBIE 2019)

Brasil (2019). Ministério da Educação. e-MEC - Sistema de Regulação do Ensino Superior. Disponível em: <http://emec.mec.gov.br>. Acesso em: 03 de março de 2019.

Brasscom. Sobram vagas em tecnologia - Crescimento do Setor Acelera. Disponível em: $<$ https://brasscom.org.br/sobram-vagas-em-tecnologia-crescimento-do-setor-acelera/>. Acesso em: 02 de junho de 2019.

Damasceno, I., \& Carneiro, M. (2018, Outubro). Panorama da Evasão no Curso de Sistemas de Informação da Universidade Federal de Uberlândia: Um Estudo Preliminar. Simpósio Brasileiro de Informática na Educação-SBIE (Vol. 29, No. 1, p. 1766).

Dejavite, L. M., Guedes, K., Beleti Jr., C. R. , Molin Filho, R. G. D., \& Santiago Jr, R. M. (2017) O desempenho dos estudantes nas disciplinas de algoritmos e programação de computadores: Um retrato dos cursos de engenharia da UFPR. XLV Congresso Brasileiro de Educação em Engenharia.

Florencio, F. G. (2015). Evasão no curso de Licenciatura em Computação da Universidade de Brasília (Trabalho de conclusão de curso). Universidade de Brasília, Brasília, DF, Brasil.

Galafassi, F. P., Gluz, J. C., \& Galafassi, C. (2013). Análise crítica das pesquisas recentes sobre as tecnologias de objetos de aprendizagem e ambientes virtuais de aprendizagem. Revista Brasileira de Informática na Educação, 21(3), 41-52.

Giraffa, M. M., \& da costa Mora, M. (2013). Evasão na disciplina de algoritmo e programação: um estudo a partir dos fatores intervenientes na perspectiva do aluno. III Conferencia Latinoamericana sobre el Abandono en la Educación Superior.

Hoed, R. M. (2016). Análise da evasão em cursos superiores: o caso da evasão em cursos superiores da área de Computação (Dissertação de mestrado). Universidade de Brasília, Brasília, DF, Brasil.

Koliver, C., Dorneles, R. V., \& Casa, M. E. (2004). Das (muitas) dúvidas e (poucas) certezas do ensino de algoritmos. XII Workshop de Educação em Computação.

Piva Jr, D., \& Freitas, R. L. (2010). Estratégias para melhorar os processos de abstração na disciplina de Algoritmos. Simpósio Brasileiro de Informática na Educação-SBIE (Vol. 1, No. 1).

Reino, L. R. A. C., Hernández-Domínguez, A., Júnior, O. D. G. F., Carvalho, V., Barros, P. A. M., \& Braga, M. (2015, October). Análise das Causas da Evasão na Educação a Distância em uma Instituição Federal de Ensino Superior. In Brazilian Symposium on Computers in Education (Simpósio Brasileiro de Informática na Educação-SBIE) (Vol. 26, No. 1, p. 91).

SBC. Sociedade Brasileira de Computação. Currículo de Referência. Disponível em: $<$ http://www.sbc.org.br/documentos-da-sbc/summary/131-curriculos-de-referencia/763curriculo-de-referencia-lic-versao-2002>. Acesso em: 31 de maio de 2019.

Stadelhofer, L. E., \& Gasparini, I. (2018, 10). Ensino de Algoritmos e Lógica de Programação para os Diferentes Cursos: Um Mapeamento Sistemático da Literatura. Anais do XXIX Simpósio Brasileiro De Informática Na Educação (SBIE 2018).

Veloso, T., \& Almeida, E. (2013). Evasão nos cursos de graduação da Universidade Federal de Mato Grosso, campus universitário de Cuiabá - um processo de exclusão. SérieEstudos - Periódico do Programa de Pós-Graduação em Educação da UCDB, 0(13). 